\title{
Long-term Outcomes of Hypofractionated Stereotactic Radiotherapy for the Treatment of Perioptic Nonfunctioning Pituitary Adenomas
}

\author{
Aiko HATA, ${ }^{1,2}$ Masaya OdA, ${ }^{1}$ Takahiro ONO, ${ }^{1}$ Akira SuZUKI, ${ }^{3}$ Noriaki HANYU, ${ }^{4}$ \\ Masataka TAKAHASHI, ${ }^{1}$ Toshio SASAJIMA, ${ }^{5}$ Manabu HASHIMOTO, ${ }^{6}$ \\ Taizen NAKASE, ${ }^{1,2}$ and Hiroaki SHIMIZU ${ }^{1,2}$
}

\author{
${ }^{1}$ Department of Neurosurgery, Akita University Graduate School of Medicine, Akita, Akita, \\ Japan \\ ${ }^{2}$ Department of Stroke Comprehensive Medical Center, Akita University Hospital, Akita, \\ Akita, Japan \\ ${ }^{3}$ Yokohama Tsuoka Noshinkeigeka, Yokohama, Kanagawa, Japan \\ ${ }^{4}$ Department of Neurosurgery, Hakodate Shintoshi Hospital, Hakodate, Hokkaido, Japan \\ ${ }^{5}$ Department of Dementia Research, Akita Prefectural Center for Rehabilitation and \\ Psychiatric Medicine, Daisen, Akita, Japan \\ ${ }^{6}$ Department of Radiology, Akita University Graduate School of Medicine, Akita, \\ Akita, Japan
}

\begin{abstract}
The efficacy of stereotactic radiotherapy (SRT) has been well established for postoperative residual and recurrent nonfunctioning pituitary adenomas (NFPAs). However, the risk of visual impairment due to SRT for lesions adjacent to the optic pathways remains a topic of debate. Herein, we evaluated the long-term clinical outcomes of hypofractionated stereotactic radiotherapy (HFSRT) for perioptic NFPAs. From December 2002 to November 2015, 32 patients (18 males and 14 females; median age 63 years; range, 36-83 years) with residual or recurrent NFPAs abutting or displacing the optic nerve and/or chiasm (ONC) were treated with HFSRT. The median marginal dose was $31.3 \mathrm{~Gy}$ (range, 17.2-39.6) in 8 fractions (range, 6-15). Magnetic resonance imaging (MRI) and visual and hormonal examinations were performed before and after HFSRT. The median follow-up period was 99.5 months (range, 9-191). According to MRI findings at the last follow-up, the tumor size had decreased in $28(88 \%)$ of 32 patients, was unchanged in $3(9 \%)$, and had increased in $1(3 \%)$. The successful tumor size control rate was $97 \%$. Visual functions remained unchanged in $19(60 \%)$ out of 32 patients, improved in $11(34 \%)$, and deteriorated in $2(6 \%)$. Two patients had deteriorated visual functions; no complications occurred because of the HFSRT. One patient developed hypopituitarism that required hormone replacement therapy. The result of this long-term follow-up study suggests that HFSRT is safe and effective for the treatment of NFPAs occurring adjacent to the $\mathrm{ONC}$.
\end{abstract}

Keywords: hypofractionated stereotactic radiotherapy, long-term outcomes, nonfunctioning pituitary adenomas, optic chiasm, optic nerve

Received November 5, 2020; Accepted March 5, 2021

Copyright@ 2021 The Japan Neurosurgical Society

This work is licensed under a Creative Commons Attribution-NonCommercial-NoDerivatives International License. 


\section{Introduction}

Pituitary adenomas represent $19.2 \%$ of primary brain tumors and approximately half of these are nonfunctioning pituitary adenomas (NFPAs). ${ }^{1)}$ Surgical removal, mainly through a transsphenoidal approach, is usually the first choice of treatment for NFPAs; however, residual or regrown tumors after surgery are often treated with radiation therapy, especially stereotactic radiotherapy (SRT). ${ }^{2-4)}$

The efficacy and safety of SRT for NFPAs have been reported. ${ }^{5-8)}$ However, SRT for lesions adjacent to the optic nerve and/or chiasm (ONC) is associated with a potential risk for radiation-induced visual disturbance. The total dose to cause radiation-induced optic neuropathy in $5 \%$ of patients in 5 years is reported to be $50 \mathrm{~Gy},{ }^{9)}$ and a single radiation dose of 8-10 Gy is tolerable for the ONC. ${ }^{10)}$ Therefore, a balance between tumor control and ONC tolerance for radiation ${ }^{10-12)}$ needs to be considered during treatment. Stereotactic radiosurgery (SRS) with a single irradiation strategy, such as usual gamma knife therapy, may have limitations; thus, fractionated stereotactic radiotherapy (FSRT) ${ }^{13-15)}$ using LINAC has been applied for near-ONC lesions, and this has a benefit of reducing the irradiation dose/shot and allows for more flexible planning of irradiation angles and fraction numbers. ${ }^{10)}$ Previous reports of FSRT demonstrated that this is safe and achieves an acceptable tumor control rate; however, a long treatment period (5-6 weeks) is required to apply 23-28 fractions of 1.8-2.0 Gy/shot to achieve a total dose of 46-50.4 Gy.

Recently, hypofractionated stereotactic radiotherapy (HFSRT) using higher dose/shot and fewer fraction numbers than FSRT has been increasingly applied for NFPAs ${ }^{5,16-18)}$ with sufficient tumor control and preservation of visual function. ${ }^{17,18)}$ However, the effect of HFSRT has not been well investigated in tumors that touch and compress the ONC.

The purpose of this study was to retrospectively assess the outcomes of patients treated with HFSRT for NFPAs which are touching to or compressing the ONC, with a special focus on long-term outcomes.

\section{Methods}

\section{Patient cohort}

A retrospective review of the HFSRT database of our hospital was performed to identify patients with NFPAs that touched or compressed the ONC, who were treated with HFSRT between 2002 and 2015. All patients with postoperative residual or recurrent NFPA who underwent the first radiation therapy at our institution were included. This study conforms to the STROBE reporting criteria for a retrospective cohort study.

In all, 32 cases were included in this study. The principal strategy at our hospital for the management of residual or recurrent tumors after first surgical removal is to repeat the surgical removal. HFSRT was performed as an alternative treatment for patients with poor general condition, elderly, or based on patient's request.

Previous surgical and medical histories for NFPA, treatment outcomes, and complications were obtained with retrospective review of the institutional medical records.

Patient demographics and HFSRT prescriptions are summarized in Table 1. According to magnetic resonance imaging (MRI) findings, tumor-ONC relationships were classified into the following two types: "touching (no deformity or misalignment of the ONC on MRI)" and "compressing (deformity and/or misalignment of the ONC on MRI)," as described in Table 1.

Institutional Review Board approval, including a waiver for informed consent, was obtained for this study.

\section{Radiosurgical technique}

HFSRT was performed with Clinac 600C (Valian Co., Palo Alto, CA USA) until 2014 and with Novalis (Brainlab Co., Tokyo, Japan) thereafter. On the day of HFSRT, a stereotactic headframe was placed on the patients by a neurosurgeon. A fusion image of high-resolution gadolinium-enhanced MRI and non-contrasted computed tomography of the brain was evaluated to plan the treatment according to the software of either machine. Planning target volume (PTV) was estimated to cover the gross tumor volume (GTV) with a $0-2 \mathrm{~mm} 3 \mathrm{D}$ margin. The marginal dose was approximately $30 \mathrm{~Gy}$, applied in 7 fractions, with variation as shown in Table 1.

The radiation dose was directed to the periphery of the lesion. Prescribed isodoses were selected individually for each patient, ideally to cover $>95 \%$ of the target volume. Selection of the total tumor dose, number of fractions, and the prescribed isodoses varied depending on the size, shape, and location of the tumor. Lenses, eyes, optic nerves, optic chiasm, and other lesions were contoured as organs at risk. Depending on these factors and the relationship between the tumor and the ONC, the irradiation area was reduced, and the threedimensional (3D) margin was adjusted in the area adjacent to the ONC. The equivalent dose for $2 \mathrm{~Gy}$ dose per fraction (EQD2) was calculated using the linear quadratic model, and the central dose was set such that the EQD2 at ONC was within $50 \mathrm{~Gy}$. 
Table 1 Patient characteristics and HFSRT prescriptions

\begin{tabular}{lc}
\hline Total & 32 \\
Median age (range), years & 63 (36-83) \\
Gender, male/female & $18 / 14$ \\
Median follow-up time (range), months & $99.5(10-191)$ \\
Postoperative residual component/postoperative re-increase & $19 / 13$ \\
Median interval between final surgery and HFSRT (range), months & 8 (1-152) \\
Median tumor volume (range), cm ${ }^{3}$ & $5.33(1.09-62.66)$ \\
Cavernous sinus infiltration (\%) & $17(53 \%)$ \\
Relationship between the tumor and ONC & $10(7)$ \\
Touching (with visual symptoms) & $22(18)$ \\
Complessing (with visual symptoms) & 1 \\
Prescribed median marginal dose (range), Gy/fraction number & 9 \\
$33.5 / 6$ & 7 \\
28.4(17.2-33.1)/7 & 3 \\
$30.8(22.1-34) / 8$ & 8 \\
$30.4(30.2-31.7) / 9$ & 1 \\
$32.9(29.6-34.3) / 10$ & 1 \\
$37.4 / 11$ & 2 \\
$35.7 / 14$ & \\
$38.5(37.4-39.6) / 15$ & \\
\hline
\end{tabular}

Touching: the tumor is touching to the ONC without deformity or displacement of the ONC. Compressing: the tumor is compressing the ONC with deformity and/or displacement of the ONC. HFSRT: hypofractionated stereotactic radiotherapy, ONC: optic nerve and/or chiasm.

Dosimetric data were obtained from the treatment planning software. The prescription dose and special relationship to ONC were recorded by taking a picture every time.

\section{Follow-up procedures}

Patients were scheduled for a follow-up that included MRI, endocrinologic, and ophthalmologic examinations every 3 months for a year after HFSRT, approximately every 6 months for the next 2 years, and every year thereafter. Ophthalmological tests to assess visual acuity and visual fields were performed when there were any changes in subjective symptoms, physical findings, or tumor enlargement on imaging. In case of any changes in clinical symptoms, patients were instructed to return as needed. Long-term follow-up data were recorded from the most recent medical record, including for one patient who died due to heart failure and six patients who were lost to follow-up.

\section{Treatment outcomes}

Treatment outcomes were evaluated in terms of changes in tumor size on MRI, as well as visual function, including visual acuity, Goldman visual field examinations, and endocrinological evaluation.

MRI was performed using a $1.5 \mathrm{~T}$ system (Signa HDxt, GE Healthcare, Chicago, IL, USA) or 3.0T system (Discovery MR 750w, GE Healthcare) with an 8-channel phased-array coil. Axial and coronal T2-weighted (repetition time/echo time, 500/14 m/s; flip angle, $90^{\circ}$; field of view, $18 \times 20.3 \mathrm{~cm}$; matrix, $256 \times 192$; slice thickness, 2.5 or $3.0 \mathrm{~mm}$; and slice interval $3 \mathrm{~mm}$ ) and T1-weighted contrast-enhanced images (repetition time/echo time, 500/14 m/s; flip angle, $90^{\circ}$; field of view, $18 \times 20.3 \mathrm{~cm}$; matrix, 256 $\times 192$; slice thickness, 2.5 or $3.0 \mathrm{~mm}$; and slice interval $3 \mathrm{~mm}$ ) were obtained. Meglumine gadopentetate (Magnevist Syringe, Bayer Schering Pharma; $0.2 \mathrm{~mL} / \mathrm{kg}$ of body weight) was used as the contrast agent. Changes in maximum tumor size by $\geq 2 \mathrm{~mm}$ on MRI were regarded as an increase or decrease in tumor size, according to our previous report. ${ }^{19)}$

Normal visual function before HFSRT was defined as corrected vision $\geq 0.7$, with no visual field deficit. An improvement in visual acuity was defined as an increase of 0.2 or more in corrected visual acuity, whereas an exacerbation of visual acuity was defined as a decrease of 0.2 or more in corrected visual 
acuity, both compared with pre-HFSRT data at follow-up. Changes in the visual field were qualitatively judged from Goldman visual field data.

Endocrinological evaluations included the level of serum adenohypophyseal hormone concentrations and their corresponding clinical symptoms. Decreased levels of hormones with corresponding symptoms were considered reflective of hypopituitarism.

\section{Statistical analysis}

The chi-square test was used to assess the differences of the treatment outcome between the "touching" and "compressing" groups. In the "touching" group, the tumor is in contact with the ONC, and in the "compressing" group, the tumor is more than $2 \mathrm{~mm}$ in pressure drainage of the ONC. Time-to-event outcomes beginning at the date of HFSRT were estimated using the Kaplan-Meier method, and the stratified outcomes between the two groups were compared using the log-rank test. The time of the last follow-up was considered to be the endpoint. $\mathrm{P}$ values $\leq 0.05$ were considered statistically significant. All statistical analyses were performed with EZR (Saitama Medical Center, Jichi Medical University, Saitama, Japan), a graphical user interface for $\mathrm{R}$ (The R Foundation for Statistical Computing, Vienna, Austria). More precisely, EZR is a modified version of $\mathrm{R}$ commander designed to add statistical functions frequently used in biostatistics. ${ }^{20)}$

\section{Results}

\section{Overall outcomes}

A total of 32 patients were included, 18 males and 14 females, with a median age of 63 years (range: 36-83 years). At the end of the median follow-up period of 99.5 months (range: 9-191 months), 28 (88\%) lesions reduced in size, 3 (9\%) lesions were unchanged, and 1 (3\%) lesion increased, resulting in a $97 \%$ success rate in the control of tumor size. Visual function (either vision or visual fields, or both) improved in 11 (34\%) patients, remained unchanged in 19 (59\%), and deteriorated in $2(6 \%)$ patients.

Seven of 32 patients required hormone replacement therapy before HFSRT, and 15 had normal pituitary function. Of the latter, one patient with normal pituitary function developed hypopituitarism, requiring hormone replacement therapy, 9 months after irradiation. This patient showed a transient increase in the cystic component of the tumor after irradiation compressing the normal pituitary gland. ${ }^{21)}$

In patients with relatively large residual tumors, defined as tumors with an approximate size $>1 \mathrm{~cm}$, HFSRT was offered when the mass had sufficiently shrunk on MRI (19 patients), and in most patients this occurred several months postoperatively. In patients with smaller residual tumors or recurrence after total resection, HFSRT was offered when regrowth/recurrence was confirmed (13 patients), and in most patients, this was more than 1 year after the first surgery.

Thus, in patients who underwent HFSRT within 12 months postoperatively, the former was mostly the case $(78 \%)$. In contrast, $79 \%$ of patients who received HFSRT 12 months or later postoperatively had the latter. The actual time interval between the first surgery and HFSRT was also influenced by the time needed for patients to consent to treatment (several weeks to several months).

\section{Outcome of visual function in patients with tumor "touching" the ONC}

Out of 10 cases with tumors "touching" the ONC (Table 2A), three cases showed normal visual function before HFSRT and seven cases had impaired vision and/or visual fields. In the latter, symptoms were due to residual visual impairment after the first surgery in six cases and due to glaucoma in one case.

After HFSRT, a reduction in tumor size was observed in eight cases and no change in two cases. Visual function was stable (eight cases) or improved (two cases), and none of the cases showed worsening during the follow-up period.

\section{Outcome of visual function in patients with tumor "compressing" the ONC}

Out of the 22 "compressing" cases (Table 2B), 17 cases showed dysfunction at the time of HFSRT in either their visual acuity or visual fields (12 cases as a sequela of the previous surgery and 5 cases due to tumor enlargement after the surgery).

After HFSRT, 20 tumors showed reduction in size, and one of each showed no change and enlargement, respectively. Visual dysfunction improved in 9 cases, remained unchanged in 11, and deteriorated due to tumor enlargement in 1 case and cataract exacerbation in another case.

The median time from surgery to irradiation for the 9 improved cases was 4 months (1-44 months) (Fig. 1A), whereas it was 14 months (3-72 months) for the 11 patients with unchanged visual function (Fig. 1B). One tumor enlargement was due to an insufficient radiation dose to a part of the tumor (Fig. 1C).

\section{Kaplan-Meier curve for tumor size changes: "touching" vs. "compressing" \\ After HFSRT, $80 \%$ of both the "touching" and "compressing” groups showed tumor shrinkage}


Table 2A Outcome of visual function after HFSRT in patients with the tumor "touching" the optic nerve and/or chiasm $(n=10)$

\begin{tabular}{lclllll}
\hline \multicolumn{2}{c}{ Visual function } & \multicolumn{2}{c}{$\begin{array}{c}\text { Changes in tumor } \\
\text { size after HFSRT }\end{array}$} \\
\hline \multicolumn{2}{c}{ Before HFSRT } & \multicolumn{3}{c}{ After HFSRT } & Reduced & 2 \\
Normal & 3 & Unchanged & 3 & Unchanged & 1 \\
& & & & Reduced & 2 \\
Decreased visual acuity & 2 & Unchanged & 1 & & \\
Visual field deficits & 1 & Unchanged & 1 & Reduced & 1 \\
Both disturbances & 4 & Unchanged & 3 & Reduced & 3 \\
& & Improved & $1^{*}$ & Unchanged & 1 \\
\hline
\end{tabular}

*Due to improved glaucoma.

Table 2B Outcomes of visual function after HFSRT in patients with the tumor compressing the optic nerve and/or chiasm $(n=22)$

\begin{tabular}{|c|c|c|c|c|c|}
\hline \multicolumn{4}{|c|}{ Visual function } & \multirow{2}{*}{\multicolumn{2}{|c|}{$\begin{array}{l}\text { Changes in tumor } \\
\text { size after HFSRT }\end{array}$}} \\
\hline \multicolumn{2}{|l|}{ Before HFSRT } & \multicolumn{2}{|c|}{ After HFSRT* } & & \\
\hline \multirow[t]{3}{*}{ Normal } & $5 * *$ & Unchanged & 4 & Reduced & 3 \\
\hline & & & & Unchanged & 1 \\
\hline & & Worsened & 1 & Enlarged & 1 \\
\hline Decreased visual acuity & 0 & - & - & - & - \\
\hline \multirow[t]{2}{*}{ Visual field deficits } & $9 * *$ & Improved & 5 & Reduced & 9 \\
\hline & & Unchanged & 4 & & \\
\hline \multirow[t]{3}{*}{ Both disturbances } & 8 & Improved & 4 & Reduced & 8 \\
\hline & & Unchanged & 3 & & \\
\hline & & Worsened & $1 * * *$ & & \\
\hline
\end{tabular}

*Four patients did not have visual acuity data after HFSRT and judged from visual field data only. ${ }^{*}$ One of these each did not have visual acuity data.

$* * *$ Due to worsened cataract.

(defined as $>2 \mathrm{~mm}$ reduction in the maximum diameter) within 50-60 months, and there was no significant difference between the groups (Fig. 2).

\section{Discussion}

In this study, we used a marginal dose of $30 \mathrm{~Gy}$ with 7 fractions as a basic plan for the treatment of NFPAs. The dose and fraction numbers were qualitatively adjusted; for large tumors or tumors compressing the ONC, the dose per fraction was reduced to $2.5 \mathrm{~Gy}$, and the number of fractions was increased up to 15 . This meticulous planning resulted in a good tumor control rate and preservation of visual function (Table 3 ).

To the best of our knowledge, no previous report has assessed the relationship between tumor-ONC compression status before HFSRT and post-treatment changes in visual function. This study divided tumor-ONC compression status into "touching" and "compressing." Seven of the 10 patients with tumors "touching" the ONC had visual dysfunction before HFSRT, which did not improve even after reduction in tumor size in five patients. We speculate that the visual dysfunction of the "touching" group patients was mostly permanent residual deficit due to initial tumor compression and/or surgical manipulation. There was no aggravation of visual function after HFSRT.

Seventeen out of 22 patients with compression had visual dysfunction before HFSRT, which improved in 9, remained unchanged in 7 cases, and worsened due to cataract in 1 case, with reduction in tumor size in every case. The remaining five patients in 
A
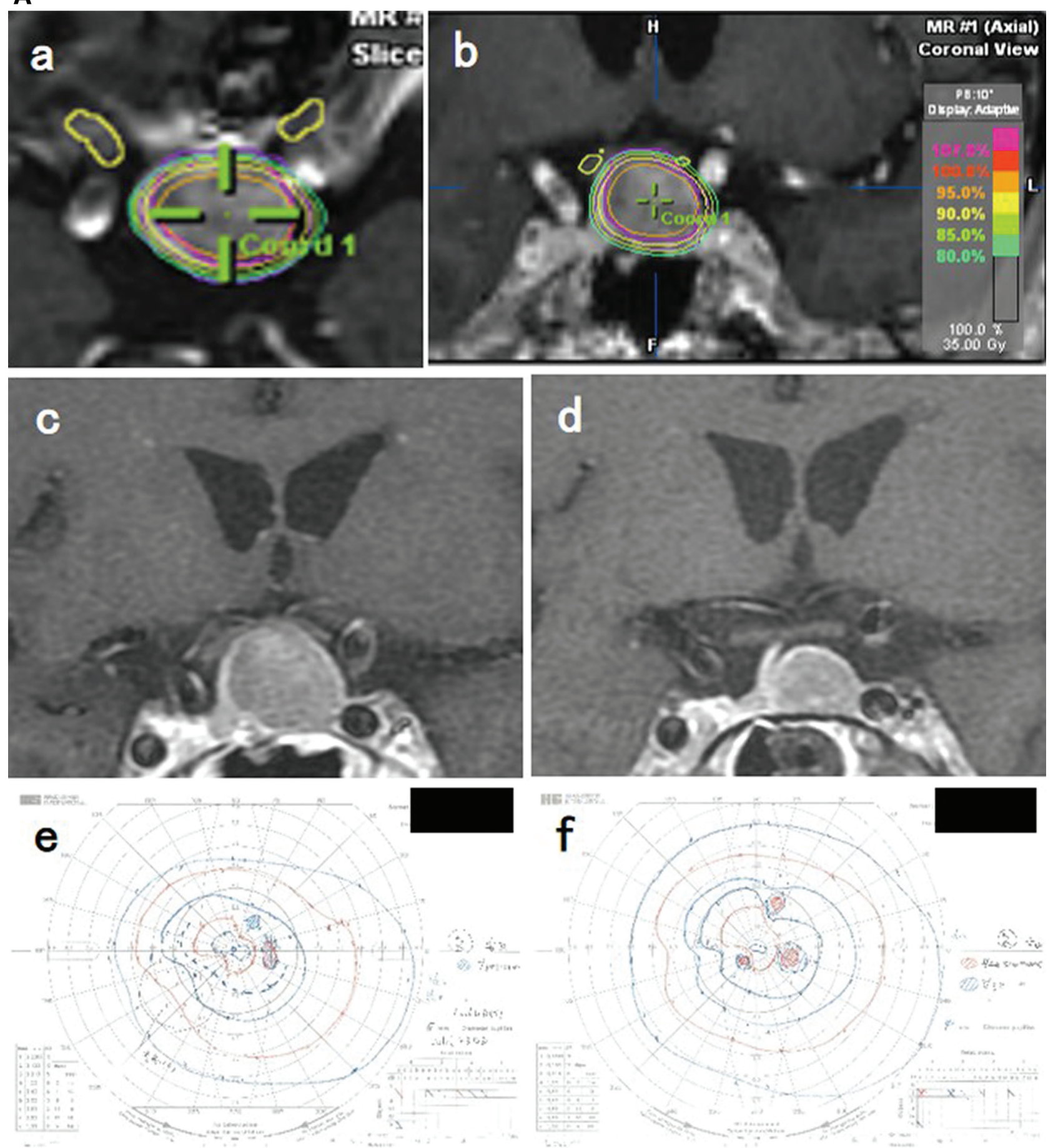

Fig. 1A Axial (a) and coronal (b) Gd-enhanced MRI superimposed with isodose distributions of HFSRT in a case of a "compressing" tumor. A 62-year-old man had bilateral inferno-lateral quadrant hemianopsia and underwent TSS in his previous hospital, with partial removal of the tumor compressing the chiasm upwards. This resulted in only partial damage due to severe fibrosis. HFSRT for the residual tumor was performed two months after surgery (GTV, $2.1 \mathrm{~mL}$; PTV, $3.0 \mathrm{~mL}$; marginal dose, $28.1 \mathrm{~Gy} / 7 \mathrm{fr}$ ). EQD2 to ONC was $47 \mathrm{~Gy}$. Gd-enhanced coronal MRIs before (c) and 9 years after (d) HFSRT showing a decreased tumor size and reduced compression of the chiasm, with normalization of his visual field impairment (e, f). EQD2: equivalent dose for $2 \mathrm{~Gy}$ dose per fraction, Gd: gadolinium, GTV: gross tumor volume, HFSRT: hypofractionated stereotactic radiotherapy, MRI: magnetic resonance imaging, PTV: planning target volume, TSS: transsphenoidal surgery. 

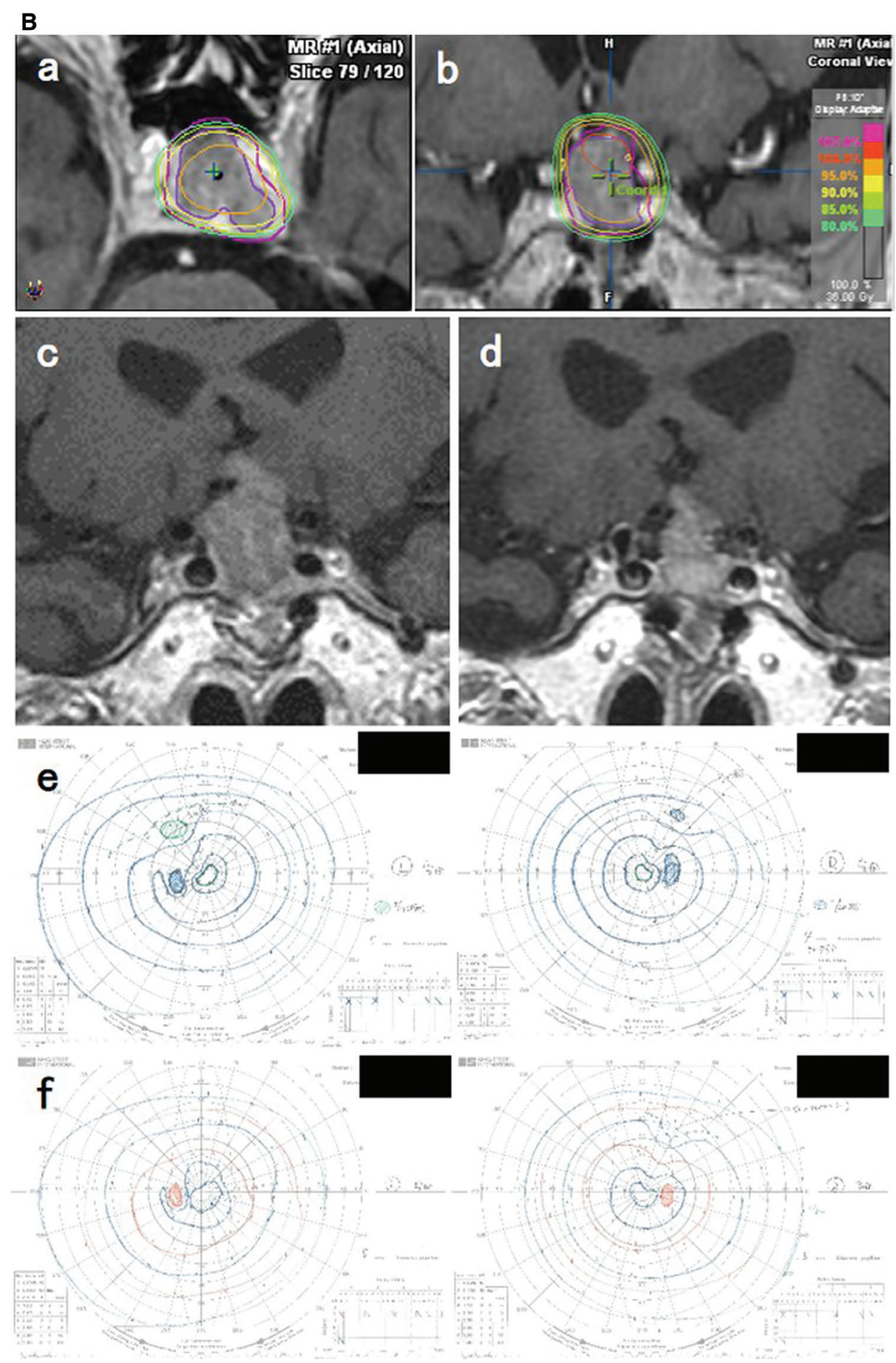

Fig. 1B Axial (a) and coronal (b) Gd-enhanced MRI superimposed with isodose distributions of HFSRT of another case of a "compressing" tumor. A 79-year-old man was blind to the left side due to cataract. Pituitary adenoma was found incidentally; however, examinations showed impaired visual acuity $(0.7)$ and inferno-lateral quadrant hemianopsia of the right eye. The tumor was partially removed through TSS. Four years later, HFSRT was performed for growing tumor (c) with $5.6 \mathrm{~mL} \mathrm{GTV}$, PTV 9.1 mL PTV, and a marginal dose of 30.2 Gy in nine fractions. EQD2 to ONC was $46.7 \mathrm{~Gy}$. Gd-enhanced coronal MRIs before (c) and 4 years after (d) HFSRT showing a decrease in tumor size, but visual field dysfunction remained unchanged (e, f). EQD2: equivalent dose for $2 \mathrm{~Gy}$ dose per fraction, Gd: gadolinium, GTV: gross tumor volume, HFSRT: hypofractionated stereotactic radiotherapy, MRI: magnetic resonance imaging, PTV: planning target volume, TSS: transsphenoidal surgery. 

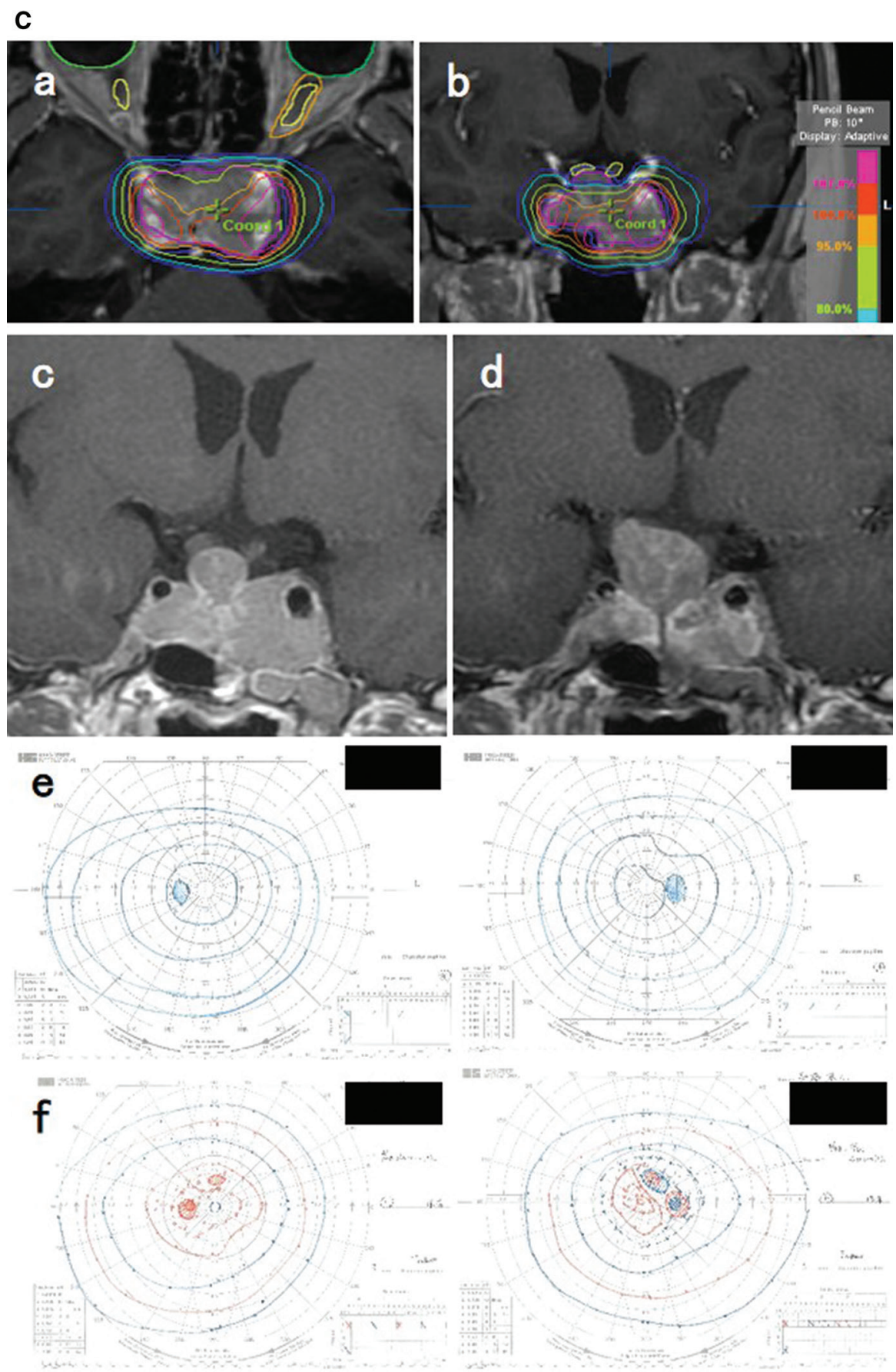

Fig. 1C Axial (a) and coronal (b) Gd-enhanced MRI superimposed with isodose distributions of HFSRT in another case of a "compressing" tumor. This 41-year-old male patient complained of diminished vision on the right side (0.2) and bilateral inferno-lateral quadrant hemianopsia. The tumor was partially removed with TSS. His visual function fully recovered with vision of 1.2; however, the residual tumor was relatively large and consent for reoperation was not obtained. HFSRT was performed for the residual tumor three months after surgery (GTV, 11.6 mL; PTV, $19.3 \mathrm{~mL}$; marginal dose, $17.2 \mathrm{~Gy} / 7 \mathrm{fr}$ ). EQD2 to ONC was $34.1 \mathrm{~Gy}$. The upper part of the tumor extended between the two optic nerves, which forced us to reduce the radiation dose to this area. Gd-enhanced coronal MRIs before (c) and 3.5 years after (d) HFSRT showing enlargement of the tumor to compress the chiasm upwards, and the patient had 1/4 right-year-side blindness (e, f). Thereafter, TSS was performed twice, and visual field dysfunction was normalized. EQD2: equivalent dose for $2 \mathrm{~Gy}$ dose per fraction, Gd: gadolinium, GTV: gross tumor volume, HFSRT: hypofractionated stereotactic radiotherapy, MRI: magnetic resonance imaging, PTV: planning target volume, TSS: transsphenoidal surgery. 
Table 3 Summary of the local controlrates from the previously reported studies of pituitary adenomas treated with HFSRT

\begin{tabular}{|c|c|c|c|c|c|c|}
\hline & \multirow{2}{*}{$\begin{array}{l}\text { No of cases } \\
(\mathrm{NF}: \mathrm{F})\end{array}$} & \multirow{2}{*}{$\begin{array}{c}\text { Median } \\
\text { follow-up } \\
\text { (range), months }\end{array}$} & \multirow{2}{*}{$\begin{array}{c}\text { Prescribed } \\
\text { median marginal } \\
\text { dose (range), Gy/ } \\
\text { fractions }\end{array}$} & \multirow{2}{*}{$\begin{array}{l}\text { Tumor } \\
\text { control } \\
\text { rate, \% }\end{array}$} & \multicolumn{2}{|c|}{$\begin{array}{l}\text { Radiation-induced late } \\
\text { adverse events }\end{array}$} \\
\hline & & & & & $\begin{array}{c}\text { Visual } \\
\text { disturbance }\end{array}$ & $\begin{array}{l}\text { Hypopituitarism } \\
\text { requiring hormone } \\
\text { replacement therapy }\end{array}$ \\
\hline Killory et al. (2009) & $20(14: 6)$ & $26.6(10.5-41)$ & $25 / 5$ & 100 & $\begin{array}{l}1 \text { transient } \\
\text { diplopia }\end{array}$ & 1 \\
\hline Iwata et al. (2011) & 100 (NF only) & $33(18-118.5)$ & $21(17-25) / 3-5$ & 98 & $\begin{array}{l}1 \text { grade } \\
2 \text { visual } \\
\text { disorder* }\end{array}$ & 0 \\
\hline Liao et al. (2014)** & $34(21: 13)$ & $36.8(16-72)$ & $21 / 3$ & 100 & $\begin{array}{l}1 \text { transient } \\
\text { diplopia }\end{array}$ & 0 \\
\hline $\begin{array}{l}\text { Puataweepong et al. } \\
\text { (2016) }\end{array}$ & $40(27: 13)$ & $37.7(14-71)$ & $25(20-35) * * * / 3-5$ & 97.5 & None & 0 \\
\hline Present study** & 32 (NF only) & $99.5(10-191)$ & $31(17-40) / 6-15$ & 97 & None & 1 \\
\hline
\end{tabular}

NF: nonfunctioning tumor, F: functioning tumor. *Common terminology criteria for adverse events(CTCAE) grade2. ** Using Novalis as the SRT system, the others using CyberKnife. ***total dose.

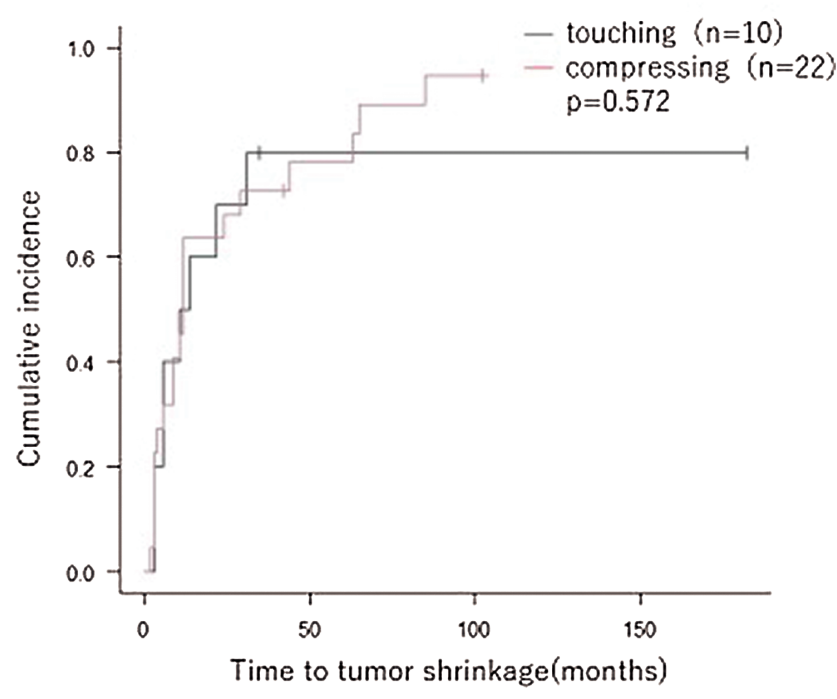

Fig. 2 A Kaplan-Meier curve for tumor size reduction (defined as more than $2 \mathrm{~mm}$ reduction of the maximum diameter) after HFSRT showing no significant difference between groups. HFSRT, hypofractionated stereotactic radiotherapy.

the "compressing" group had normal visual function before HFSRT, which remained unchanged after treatment. No aggravation of visual function due to HFSRT was again observed.

The reduction rate in tumor size of the "compressing" group was $91 \%$, similar to that of the "touching" group. In general, there is a risk of visual aggravation when a tumor compresses the ONC. ${ }^{14)}$ Our results indicate no difference between the "touching" and "compressing" groups in the long-term outcomes in terms of visual function as well as tumor control rate, pointing to the superior effect and safety of HFSRT.

Of the nine patients in the "compressing" group who showed improved visual function, eight underwent HFSRT within 10 months of their surgery. In contrast, all seven patients of the "compressing" group, who showed no change of visual function, underwent HFSRT more than 12 months after their surgery. This suggests that in cases of compression, the duration of compression may influence recovery after HFSRT. In a report using gamma knife for NFPA, patients submitted to gamma knife treatment within 6 months after surgery showed a lower complication rate and similar tumor control rate than those treated later than 6 months. ${ }^{6,7)}$ Whether or not early HFSRT is superior to late HFSRT warrants further investigation.

A reduction in tumor size, observed in most cases in this study, regardless of the relationship with the ONC, occurred within 50 months after HFSRT and reached plateau levels within 100 months. Therefore, long-term follow-up research should extend beyond 50 months to evaluate tumor reduction in tumor size outcomes.

The limitations of this study include its small number of cases, especially when divided into two groups, as well as its retrospective design. In addition, the effect of irradiation on visual functions was also difficult to evaluate in detail, because many patients in this study had visual dysfunction before HFSRT and its duration and severity were 
widely different between patients. Further prospective studies with a relatively large patient cohort are required for the validation of our results.

\section{Conclusion}

HFSRT for residual or recurrent NFPAs adjacent to the ONC is an effective and safe treatment strategy with a high tumor control rate and excellent postoperative preservation of visual functions. Further long-term follow-up research is warranted to demonstrate the long-term safety and efficacy of HFSRT.

\section{Acknowledgments}

We would like to thank the doctors, technicians, and other staff of the Radiation Therapy Department of Akita University Hospital for their cooperation.

\section{Conflicts of Interest Disclosure}

The authors have no financial relationships to disclose.

\section{References}

1) Killory BD, Kresl JJ, Wait SD, Ponce FA, Porter R, White WL: Hypofractionated CyberKnife radiosurgery for perichiasmatic pituitary adenomas: early results. Neurosurgery 64: A19-25, 2009

2) Liao HI, Wang CC, Wei KC, et al.: Fractionated stereotactic radiosurgery using the Novalis system for the management of pituitary adenomas close to the optic apparatus. J Clin Neurosci 21: 111-115, 2014

3) Barber SM, Teh BS, Baskin DS: Fractionated stereotactic radiotherapy for pituitary adenomas: single-center experience in 75 consecutive patients. Neurosurgery 79: 406-417, 2016

4) Minniti G, Traish D, Ashley S, Gonsalves A, Brada M: Fractionated stereotactic conformal radiotherapy for secreting and nonsecreting pituitary adenomas. Clin Endocrinol (Oxf) 64: 542-548, 2006

5) Colin P, Jovenin N, Delemer B, et al.: Treatment of pituitary adenomas by fractionated stereotactic radiotherapy: a prospective study of 110 patients. Int J Radiat Oncol Biol Phys 62: 333-341, 2005

6) Sun S, Liu A, Zhang Y: Long-term follow-up studies of gamma knife radiosurgery for postsurgical nonfunctioning pituitary adenomas. World Neurosurg S1878-8750: 30078-30086, 2019

7) Pomeraniec IJ, Kano H, Xu Z, et al.: Early versus late Gamma Knife radiosurgery following transsphenoidal surgery for nonfunctioning pituitary macroadenomas: a multicenter matched-cohort study. J Neurosurg 129: 648-657, 2018
8) The Committee of Brain Tumor Registry of Japan. Brain tumor registry of Japan (2005-2008). Neurol Med Chir (Tokyo) 57 Supplement-1: 9-102, 2017

9) Emami B: Tolerance of normal tissue to therapeutic radiation. Rep Radiother Oncol 1: 35-48, 2013

10) Stafford SL, Pollock BE, Leavitt JA, et al.: A study on the radiation tolerance of the optic nerves and chiasm after stereotactic radiosurgery. Int J Radiat Oncol Biol Phys 55: 1177-1181, 2003

11) Mayo C, Martel MK, Marks LB, Flickinger J, Nam J, Kirkpatrick J: Radiation dose-volume effects of optic nerves and chiasm. Int J Radiat Oncol Biol Phys 76: S28-35, 2010

12) Girkin CA, Comey CH, Lunsford LD, Goodman ML, Kline LB: Radiation optic neuropathy after stereotactic radiosurgery. Ophthalmology 104: 1634-1643, 1997

13) Gopalan R, Schlesinger D, Vance ML, Laws E, Sheehan J: Long-term outcomes after Gamma Knife radiosurgery for patients with a nonfunctioning pituitary adenoma. Neurosurgery 69: 284-293, 2011

14) Puataweepong P, Dhanachai M, Hansasuta A, et al.: The clinical outcome of hypofractionated stereotactic radiotherapy with cyberknife robotic radiosurgery for perioptic pituitary adenoma. Technol Cancer Res Treat 15: NP10-NP15, 2016

15) Kanda Y: Investigation of the freely available easy-touse software 'EZR' for medical statistics. Bone Marrow Transplant 48: 452-458, 2013

16) Höybye C, Grenbäck E, Rähn T, Degerblad M, Thorén M, Hulting AL: Adrenocorticotropic hormone-producing pituitary tumors: 12 - to 22-year follow-up after treatment with stereotactic radiosurgery. Neurosurgery 49: 284-291; discussion 291-292, 2001

17) Sheehan JP, Starke RM, Mathieu D, et al.: Gamma Knife radiosurgery for the management of nonfunctioning pituitary adenomas: a multicenter study. $J$ Neurosurg 119: 446-456, 2013

18) Iwata H, Sato K, Tatewaki K, et al.: Hypofractionated stereotactic radiotherapy with CyberKnife for nonfunctioning pituitary adenoma: high local control with low toxicity. Neuro Oncol 13: 916-922, 2011

19) Suzuki A, Hanyu N, Sasajima T, et al.: Results of fractionated stereotactic radiotherapy for the treatment of nonfunctioning pituitary adenomas. Stereotactic Radiother 15: 85-94, 2011

20) Petrovich Z, Yu C, Giannotta SL, Zee CS, Apuzzo ML: Gamma knife radiosurgery for pituitary adenoma: early results. Neurosurgery 53: 51-59; discussion 59-61, 2003

21) Izawa M, Hayashi M, Nakaya $K$, et al.: Gamma knife radiosurgery for pituitary adenomas. J Neurosurg 93: 19-22, 2000

Corresponding author: Aiko Hata, MD

Department of Neurosurgery, Akita University Graduate School of Medicine, 1-1-1 Hondo, Akita, Akita 010-8543, Japan.

e-mail: aiko_h_1226@med.akita-u.ac.jp 